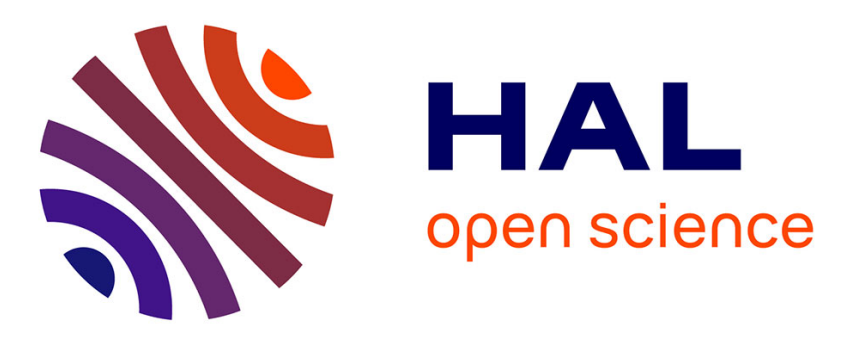

\title{
Ultrathin acoustic absorbing metasurface based on deep learning approach
}

Krupali Donda, Yifan Zhu, Aurélien Merkel, Shi-Wang Fan, Liyun Cao, Sheng Wan, B. Assouar

\section{- To cite this version:}

Krupali Donda, Yifan Zhu, Aurélien Merkel, Shi-Wang Fan, Liyun Cao, et al.. Ultrathin acoustic absorbing metasurface based on deep learning approach. Smart Materials and Structures, 2021, 30 (8), pp.085003. 10.1088/1361-665X/ac0675 . hal-03412446

\section{HAL Id: hal-03412446 https://hal.science/hal-03412446}

Submitted on 3 Nov 2021

HAL is a multi-disciplinary open access archive for the deposit and dissemination of scientific research documents, whether they are published or not. The documents may come from teaching and research institutions in France or abroad, or from public or private research centers.
L'archive ouverte pluridisciplinaire HAL, est destinée au dépôt et à la diffusion de documents scientifiques de niveau recherche, publiés ou non, émanant des établissements d'enseignement et de recherche français ou étrangers, des laboratoires publics ou privés. 


\title{
Ultrathin Acoustic Absorbing Metasurface Based on Deep Learning Approach
}

Krupali Donda, Yifan Zhu, Aurélien Merkel, Shi-Wang Fan, Liyun Cao, Sheng Wan, Badreddine Assouar*

Université de Lorraine, CNRS, Institut Jean Lamour, Nancy, France

\begin{abstract}
Acoustic metasurface has become one of the most promising platforms for manipulating acoustic waves with the advantage of ultra-thin geometry. The conventional design method of acoustic metasurface relies on numerical, trial-and-error methods to retrieve effective properties of the locally resonant unit cells. It is often inefficient and requires significant efforts to investigate the enormous number of possible structures with different physical and geometric parameters, which demands huge computational resources. This is especially when modeling narrow cavities where thermoviscous loss has to be considered. In this paper, a deep learningbased acoustic metasurface absorber modeling approach is introduced to significantly reduce the characterization time while maintaining accuracy. Based on a convolution neural network $(\mathrm{CNN})$, the proposed network can model wide absorption spectrum response in the timescale of milliseconds. The performance of the implemented network is compared with other classical machine learning methods. Using $\mathrm{CNN}$, we have demonstrated an ultrathin metasurface absorber having perfect absorption at an extremely low frequency of $38.6 \mathrm{~Hz}$ with an ultrathin thickness down to $\lambda / 684(1.3 \mathrm{~cm})$. The total path length for the propagating waves inside the channel is about $\lambda / 5.7$ which breaks the quarter-wavelength resonator theory. The network prediction is validated using the experiments to demonstrate the effectiveness of this physical mechanism. Furthermore, we propose a broadband low-frequency metasurface absorber by coupling unit cells exhibiting different properties based on the supercell concept. This approach is attractive for applications necessitating fast on-demand design and optimization of a metasurface acoustic absorber.
\end{abstract}

Keywords: Acoustic metasurfaces; Sound absorption; Deep learning; Convolutional neural network; Low-frequency; Acoustic metamaterials

*Corresponding author: badreddine.assouar@univ-lorraine.fr 


\section{Introduction}

The emergence of acoustic metamaterials [1-3] and metasurfaces [4-7] has significantly broadened the research field of acoustics, providing many fascinating ways of controlling acoustic waves propagation, such as negative refraction [8], deep subwavelength focusing beyond the diffraction limit $[9,10]$ and perfect absorption with compact structures [11]. As sound absorption is one of the most important applications in acoustics, perfect acoustic metasurface absorbers [11-18] have gained considerable attention for suppressing audible sound as excessive noise exposure is a major public health concern. The metasurface design contains locally resonant units along with highly enhanced local acoustic intensity inside each unit, which broadened the way to realize a high absorption efficiency. These resonant units are of particular interest for sound absorption in the low-frequency regime $(<100 \mathrm{~Hz})$, where the perfect absorption of sound is still a challenge due to the inherently weak intrinsic dissipation of conventional materials at this frequency range [18].

An acoustic metasurface absorber is generally based on a single or hybrid resonant system. For example, Helmholtz resonators [19], periodic groove structures [20], panels of Schroeder diffusers combined with perforated plates [21], and sonic crystals slabs with resonant scatterers [22] show good sound absorption properties in the low-frequency range. To design these acoustic absorbers, various reliable and efficient modeling tools are being used and deeply investigated. One approach is to develop analytical models using phased array methods [23]. By introducing a complex frequency plane of reflection coefficients [24] where acoustic wave theory is the design basis, designers are required to have strong expertise in this field. It prevents layman users from designing metasurface according to actual demands. Besides, a widely adopted way in acoustic metasurface design is to utilize iterative numerical full-wave simulations like finite-element method (FEM), using the commercial software COMSOL Multiphysics. Here, the all-over design time depends on the simulation time of each trial design and the number of design degrees of freedom (DOFs). The thermoacoustic module is used to solve acoustic problems in COMSOL Multiphysics which considers the effect of thermoviscous loss, particularly in confined spaces/cavities, where the complex interaction of sound with the viscous and thermal boundary layers must be accounted for. To solve such acoustic models, the thermoviscous module considers the acoustic pressure, velocity field, and variations in temperature that add many degrees of freedom [25]. It also considers a comparatively finer mesh since there are so many small-scale mechanisms involved. For these reasons, solving acoustic problems using this approach is computationally expensive. It 
provides accurate device response predictions but it is severely time-consuming especially for the low-frequency three-dimensional (3D) calculations. This is because, at low frequency $(<50 \mathrm{~Hz})$, the narrow bandwidth requires sufficiently high resolution to sweep the frequencies for accurate calculations. It then is a challenge to use a generic method to solve conveniently the novel acoustic problems [26]. Another acoustic metasurface design approach being widely studied is based on optimization strategies $[27,28]$. Based on the concept of the adjoint variable method, these methods can quickly generate non-intuitive metasurface designs in less time compared to direct brute-force searching approaches. However, their local optimizers still rely on simulation software for verifications and can be computationally expensive, depending on the performance of initial distributions of the generated metasurface designs [29].

To overcome these obstacles, we consider a data-driven approach based on machine learning methods. In recent decades, machine learning (ML) has emerged as a powerful computational tool that has been broadly applied to efficiently solve numerous scientific problems [30-36]. It offers a solution to significantly reduce time-consuming calculations with limited computational resources. Among all ML methods, deep learning has emerged as a very promising one for solving non-institutional problems [29]. It allows computational models that are composed of multiple hidden layers to intelligently learn representations of data by using the backpropagation algorithm, which can be used to represent complicated functions according to the universal approximation theorems [38-41]. Inspired by this, several deep learning-based architectures that connect electromagnetic metasurface structures to their EM responses are developed and then trained with a large amount of pre-simulated data calculated by full-wave simulations $[42,43]$. The reported studies to date mostly emphasize solving electromagnetic problems, while robust deep learning models for acoustic problems are yet to be developed $[44,45]$.

In this research, we introduce a deep learning model based on two-dimensional CNN (2D $\mathrm{CNN}$ ) as a practical tool to model acoustic metasurface absorber for low-frequency applications. Two-dimensional CNNs [40] take advantage of the spatial information and translational invariance which make them more suitable to extract relevant information at a low computational cost compared to the fully connected neural networks [31]. Moreover, the implemented metasurface absorber structure solves two key challenges of the previous works $[11,17]$. The first one is to remove the dependency of absorption on the length of the coiled channel [11]. In a coiled metasurface, the length of the coiled channel strictly requires quarter wavelength channel length to achieve perfect absorption, creating difficulties in adjusting the 
absorption frequency and bandwidth. As a solution to this problem, a multi-coiled metasurface with an embedded aperture and the labyrinthine structure was designed [17]. Second, one doesn't need to create a specific design of the channel for acoustic wave propagation to tune the desired resonance frequency.

In our approach and method, we connect the metasurface structure with its acoustic properties through deep learning methods, where we set up and train the deep learning model by a set of samples; the model is capable of finding out the inner rules between metasurface structure and its acoustic properties. We have compared the performance of our implemented technique with other classical machine learning algorithms. Using the $\mathrm{CNN}$, as we will demonstrate, full absorption at $38.6 \mathrm{~Hz}$ with a total thickness down to $\lambda / 684$ is achieved. The total path for the propagating waves inside the channel is calculated by the mean distance through the channel. It is worth mentioning that this path is about $\lambda / 5.7$ which is significantly smaller than the one allowed by the classical quarter-wavelength resonators.

The proposed design of the absorbing acoustic metasurface we are reporting here not only deals with the sound absorption, but potentially could be useful to produce other functionalities. Indeed, one of the idea this smart design could lead to is the heat energy harvesting that could be collected from the absorbed acoustic energy. The latter is usually lost as a heat, so one can consider an additional system formed by thermoelectric and piezoelectric materials to tackle on this aspect by collecting and converting the absorbed acoustic energy to electric current.

\section{Theory and design}

Based on 2D convolutional neural networks, an efficient ultrathin metasurface modeling method is proposed. It aims to uncover the hidden relationship between metasurface models and their absorption spectral responses and thus accurately predict responses for given metasurface designs. In this section, we first introduce the structure of the metasurface, followed by the overall design idea beginning from the aspect of training and generating spectral responses. Here, the propagation channel length is used to tune the resonance frequency. The longer the channel length it is, the lower the resonance frequency it gets.

\subsection{Structure of acoustic metasurface absorber}

The geometry of the metasurface, as shown in Fig. 1, consists of a resonant absorber system composed of a cavity, with some random coiled pattern inside, covered by a plate of thickness 
$\mathrm{t}=2 \mathrm{~mm}$ with a circular opening. In our study, we used a fixed periodicity of the unit cell $\mathrm{a}=100 \mathrm{~mm}$. The thickness of the outer walls is $2 \mathrm{~mm}$, therefore the lateral dimension of the square cavity is $96 \mathrm{~mm}$. The cavity is decomposed into square lattice sites of $2 \times 2 \mathrm{~mm}^{2}$ to create the propagation channel with a wall thickness of $2 \mathrm{~mm}$. The lattice sites are labeled as $r_{1,1}, r_{1,2}, \ldots, r_{48,48}$. It can be presented as a $48 \times 48$ lattice mesh marked with '.' or blank. Blank lattice site means that area is filled up by air, and '.' lattice site means the area is filled up with PLA (density, $\rho=2700 \mathrm{~kg} / \mathrm{m}^{3}$ ). The total lattice mesh is then encoded into a pattern matrix $a$,

$$
a=\left[\begin{array}{ccc}
r_{1,1} & \cdots & r_{1,48} \\
\vdots & \ddots & \vdots \\
r_{48,1} & \cdots & r_{48,48}
\end{array}\right]
$$

where $r_{i, j}=0$ and 1 if the lattice site is filled with air and PLA, respectively.

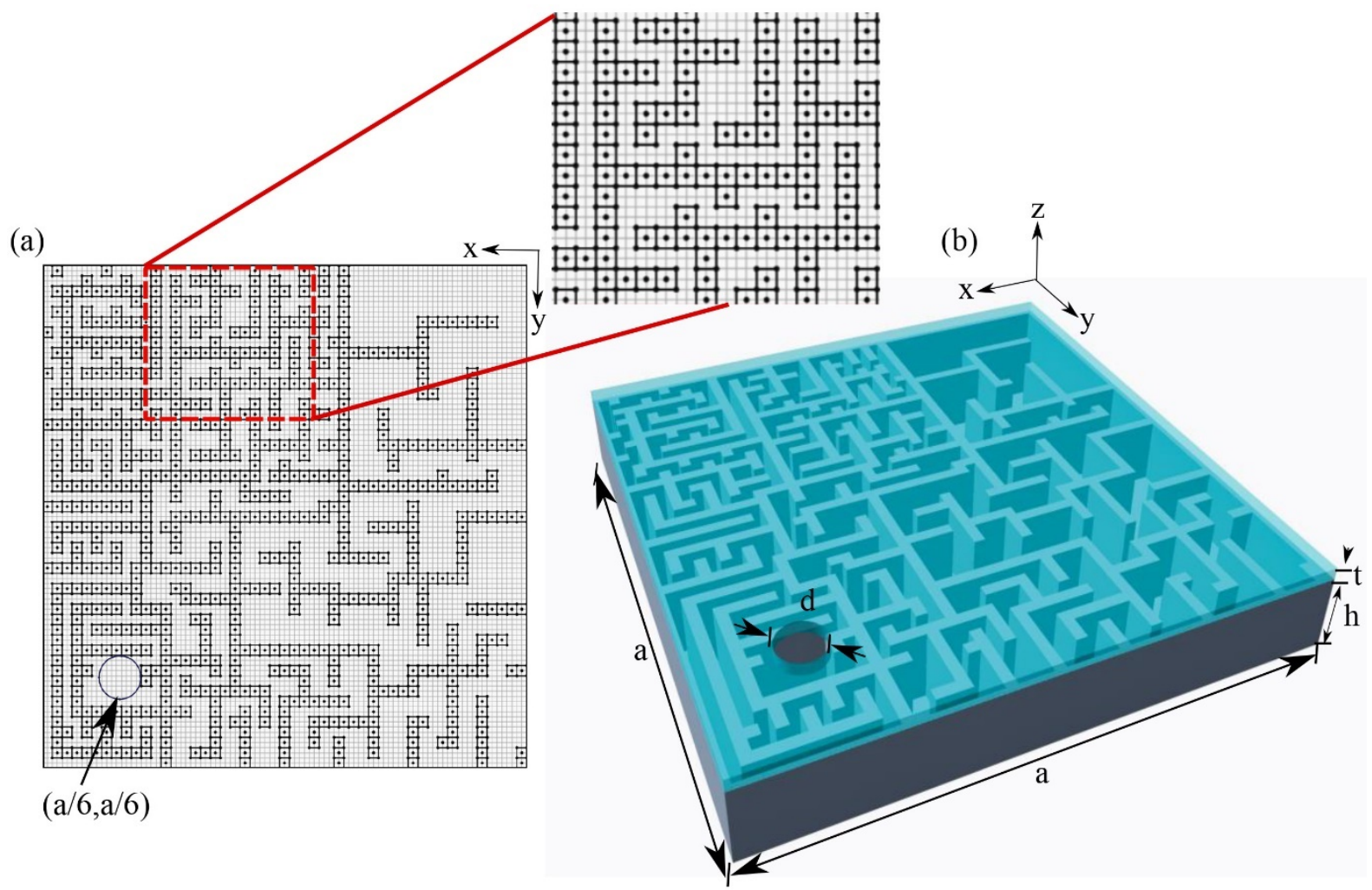

FIG. 1. Schematic illustration of (a) matrix encoding and (b) metasurface absorber (crossarea $=\mathrm{a} \times \mathrm{a}$ ). The normal incident wave propagates along the $\mathrm{z}$-direction and penetrates into the channel from the through-hole the perforated plate.

Here, each pattern matrix corresponds to a set of absorption coefficients. For our case, the total number of lattice sites is 2304 . The possible numbers of pattern matrices are $2^{2304}$. Using such matrices, large numbers of free form channels can be created. Ideally, it takes millions of years for the calculation of all data, which is an impossible task under current calculation conditions. 
Therefore, the use of deep learning networks is advantageous here to find optimum value while reducing computational time.

\subsection{Training process}

To get the training data sets, metasurface pattern matrices are regarded as input data while the absorption coefficients are taken as the output data. To obtain the absorption coefficients of the metasurface absorber, simulations are conducted with the preset thermoviscous module of COMSOL Multiphysics v5.5. Sound hard boundaries are imposed on the interfaces between air and solid due to the huge impedance mismatch between air and solid materials. A normal incident plane wave with unit amplitude propagates along the z-direction. Here, the unit cell

itself acts as a metasurface. The absorption coefficient, $\alpha$, can be expressed as $\alpha=1-|r|^{2}$, with $\mathrm{r}=(\mathrm{Z}-1) /(\mathrm{Z}+1)$ representing the complex reflection coefficient and $\mathrm{Z}$ is the normal acoustic impedance.

We generate 10,000 pairs of absorption coefficients and metasurface pattern matrices to form our dataset. We take $80 \%$ of the dataset as a training set and the rest $20 \%$ as the testing set. Each pattern matrix is subjected to a sound wave of a given frequency range and we obtain the value of the absorption coefficient at each frequency. Thus, each pattern matrix is corresponding to a set of absorptions that form a continuous absorption spectrum for the given frequency band. Normally, in terms of the volume of training data in deep learning, we care more about the ratio between the number of samples and the number of features for each sample rather than the number of samples only.

\subsection{Network Architecture and Results}

The overall 2D CNN design we use is illustrated in Fig.2. The objective of the training is to achieve total absorption at low frequencies while keeping the lateral dimension and the depth of the metasurface unchanged. We train the network using Adam-optimizer with a mean squared error loss function. Here, the neural architecture consists of two 2D convolution layers along with two max-pooling layers. In our case, the shape of the input layer is $(48,48,1)$. The network starts with a convolution layer named as $h^{(1)}$. The convolution layers are used to extract the latent features from the input as illustrated in Fig.2. Each convolution layer consists of a set of filters, which are applied over the input to the layer. The operation of the 1D convolution layer with the filter size $(4,1)$ can be explained by the schematic as illustrated in Fig.3 which can be easily extended to $2 \mathrm{D}$. Here, the neurons $r_{1}, r_{2}, r_{2}, \ldots, r_{n}$ in the input layer 
indicate the features of the input. For our 2D CNN, the stride of $(2,2)$ and the filters of the size $(2,2)$ are applied on the input layer which slides over the input features. Here, we use a total of 128 filters. The output of the $h^{(1)}$ layer is fed to the max pool layer $h^{(2)}$. This layer downsamples the input representation, reducing its dimensionality. Here, we use a pool size of $(2,2)$ and the stride of $(2,2)$. As such, there is a significant reduction in the number of parameters or weights. This phenomenon lowers the computational cost and mitigates over-fitting. Similarly, another convolution layer $h^{(3)}$ is used which followed by a pooling layer, $h^{(4)}$ with same filter size. The bias " $\mathrm{b}$ " is added over every output of the convolution operation. A non-linear activation function using the Rectified Linear Units (ReLU) is applied to each layer.

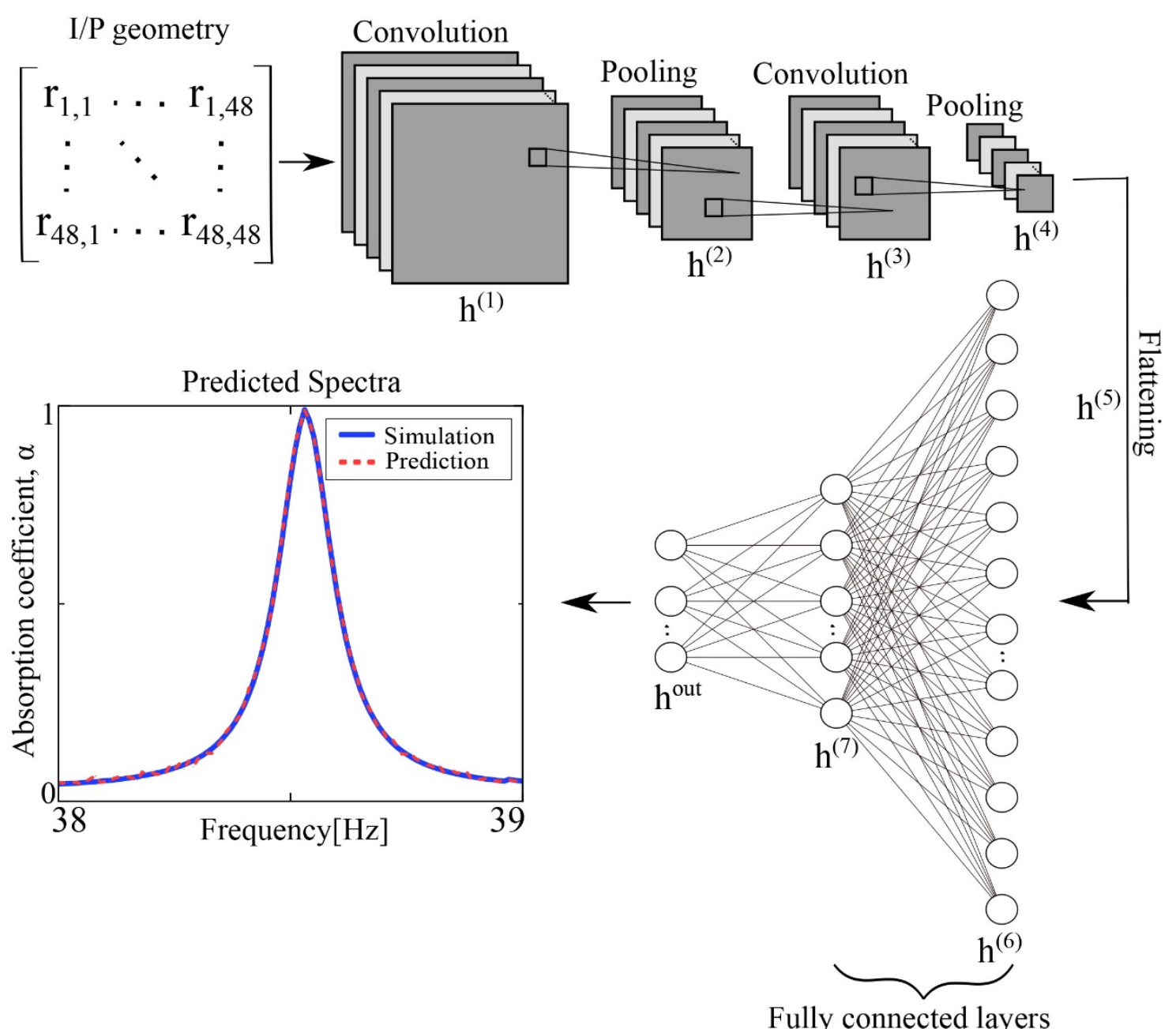

FIG. 2. An illustration of the deep learning network architecture for the metasurface absorber design. A set of geometric inputs is fed to $\mathrm{CNN}$ which makes data smoothed and upsampled in a learnable manner. The output of $\mathrm{CNN}$ is fed to the fully connected layers which produce a predicted spectrum (red dashed curve) compared to the ground truth (blue curves). 
The output of the pooling layer is then flattened using a flattening layer, $h^{(5)}$ and is passed through two fully connected layers, $h^{(6)}$ and $h^{(7)}$ of sizes DS $=\{500,100\}$. The output of the fully connected layer $h^{(7)}$ is calculated by,

$$
h_{j}^{(7)}=\sum_{i=1}^{500} w_{j i}^{h^{(7)}} h_{i}^{(6)}+b_{j}^{(7)}
$$

where, $w_{j i}^{h^{(7)}}$ is the trainable matrix corresponds to the layer $h^{(7)}$. i and $\mathrm{j}$ represent the neurons in layers $h^{(6)}$ and $h^{(7)}$ respectively. The bias term, $b_{j}^{7}$ represents the bias associated with a neuron $\mathrm{j}$ present in the layer $h^{(7)}$.

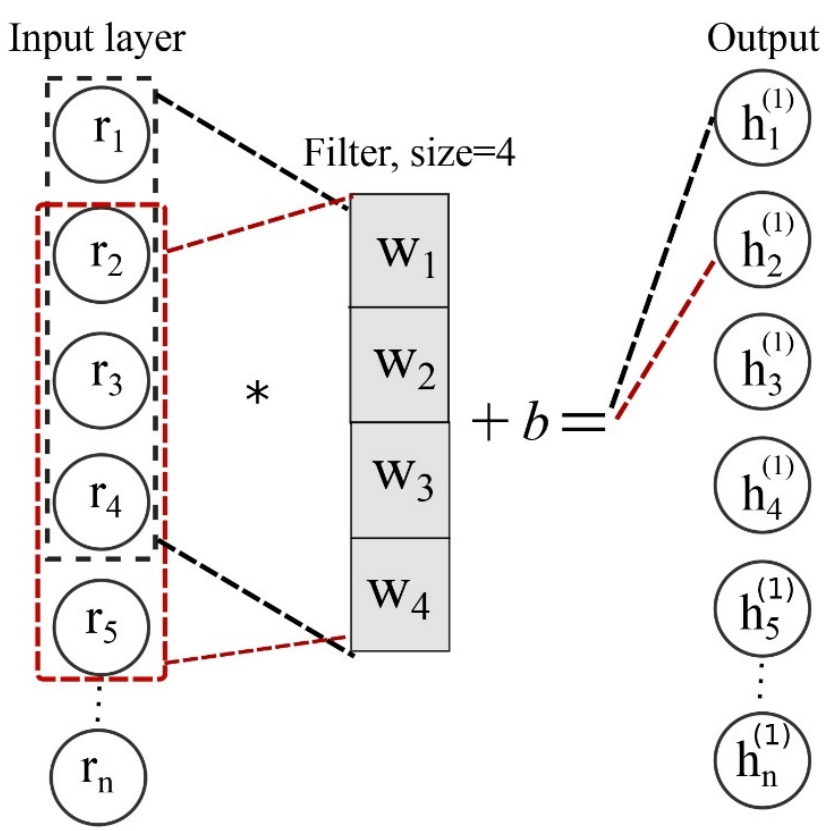

FIG. 3. Schematic of the 1D convolution operation.

Finally, the sigmoid function is used to obtain the predicted output from the model. Here, $h^{\text {out }}$ represents the absorption coefficients for the given frequency range. Without loss of generality, the spectra of interest were set to be from 30 to $70 \mathrm{~Hz}$. The proposed model is built under the windows 10 operating system. The configuration of the computer is Intel(R) Core (TM) i53470 CPU@3.19GHz. It is realized on the Anaconda platform with python version 3.6. Keras framework with a TensorFlow backend is also used to set up the model (See the appendix for the hyperparameters). Here, the mean squared error (MSE) is used as a loss function. We predict the absorption spectrum for testing data using the trained network and compare the results with those obtained by the COMSOL Multiphysics. Fig. 4 depicts the spectral response of four representative cases from the testing data that clearly indicate the predicted frequencydependent absorption spectra (red dashed curve) match the simulated spectra (blue curves) well 
for different curves. The predicted absorption spectra $\left(\alpha_{p}\right)$ and simulated absorption $\left(\alpha_{s}\right)$ overlap almost perfectly such that we plot, on the right axis (shaded dark yellow area), the absolute value of the difference in absorption, defined as $\left|\alpha_{s}-\alpha_{p}\right|$. More importantly, once trained with enough data, the proposed 2D CNN can generate the predictions in milliseconds, which is four orders of magnitude faster than conventional FEM based software. This makes it an attractive substitute for conventional simulation tools for applications such as highthroughput device design and optimization.

(a)

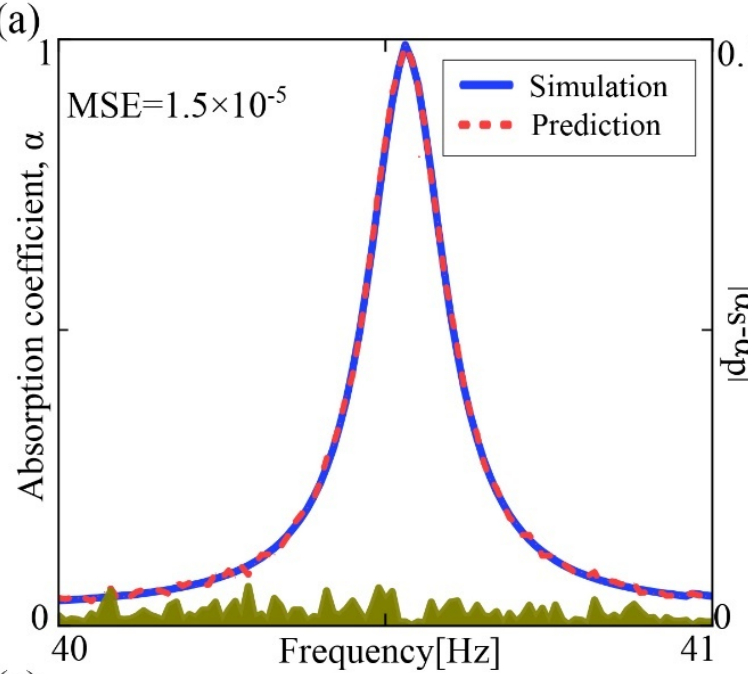

(c)

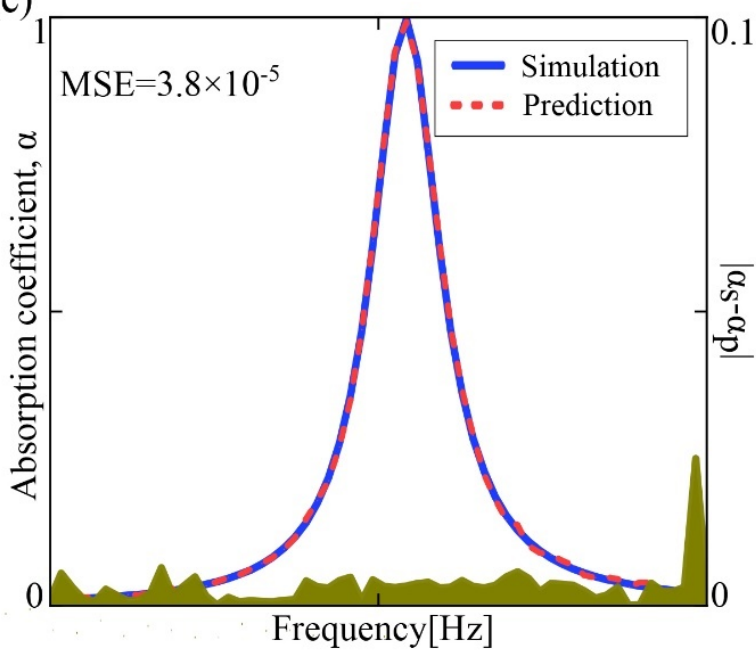

(b)

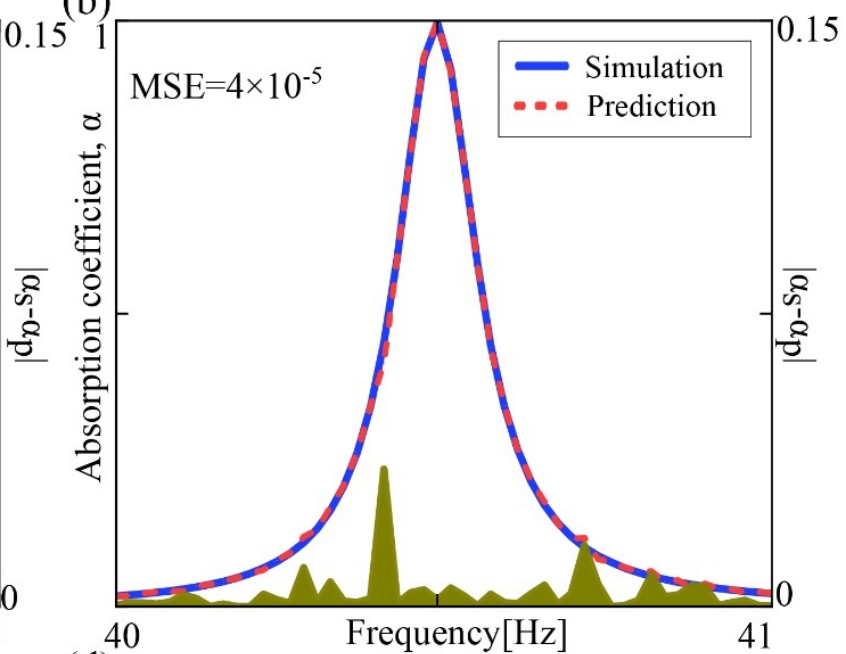

(d)

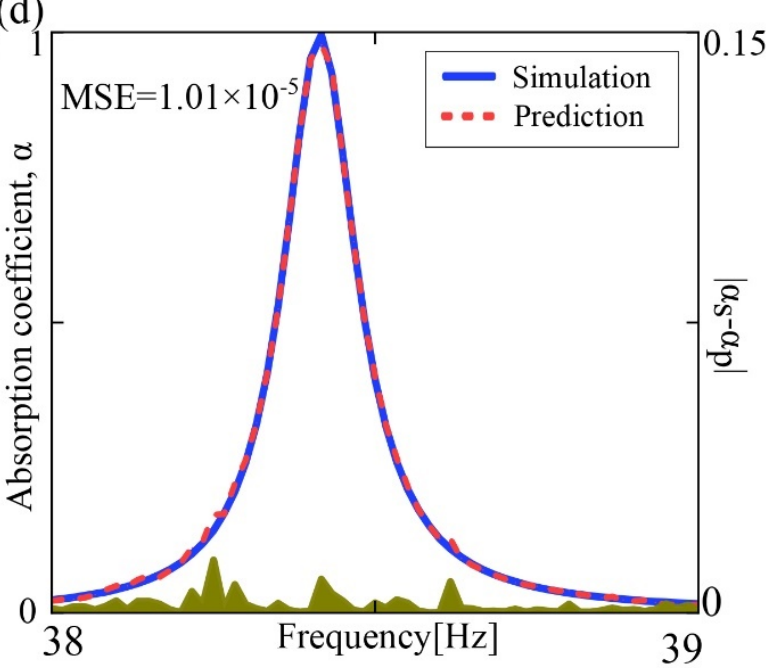

FIG. 4. Examples of network predictions of the frequency dependent absorption (red dashed curve) and simulated spectra (blue curves) demonstrating excellent prediction accuracy for a variety of input geometries. The shaded dark yellow area shows the absolute value of the difference in predicted and simulated absorption, i.e. $\left|\alpha_{s}-\alpha_{p}\right|$, shown on the right vertical axis.

For the further analysis of the metasurface absorber, we simulate the pressure profile at the resonance frequency $38.6 \mathrm{~Hz}$ as shown in Fig. 5 (corresponding absorption curve is shown 
in Fig.2). It can be observed that the acoustic pressure is higher in deeper regions of the structure at the resonance case, whose distribution is similar to previous metasurface absorber designs with a gradient channel $[16,17]$. When the wave passes through the channel at the coupling resonant frequency, it is being attenuated and gets absorbed due to thermoviscous loss.

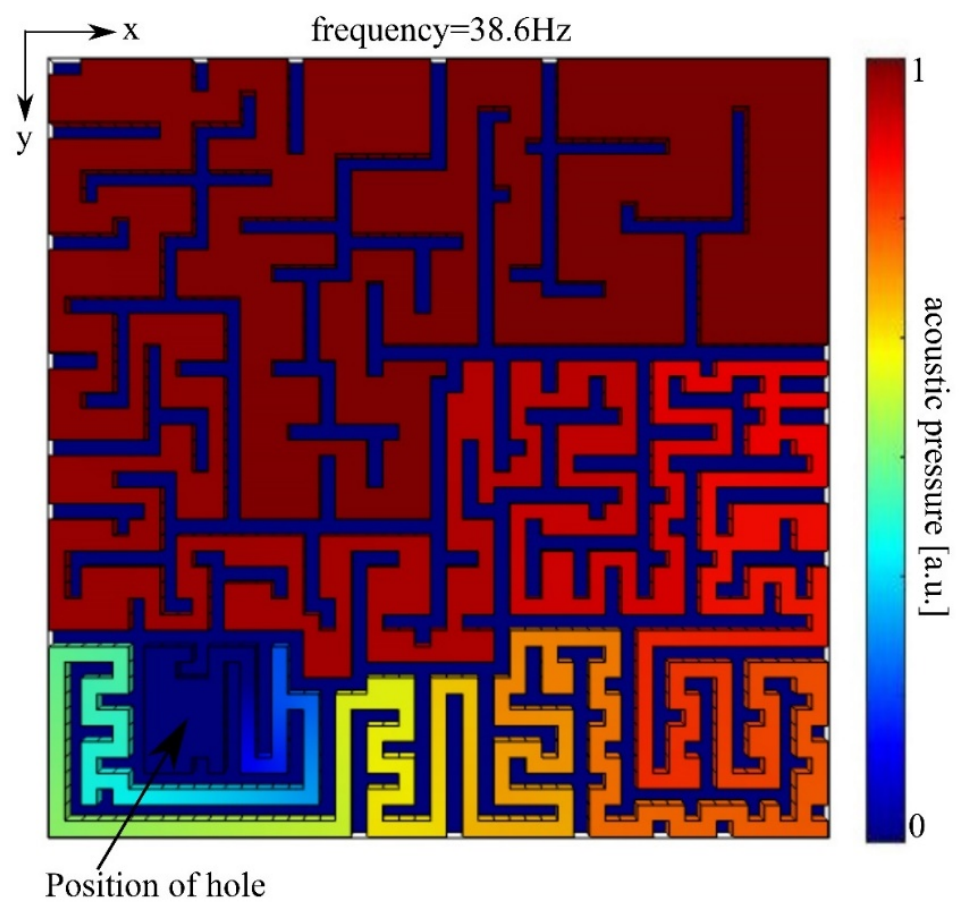

FIG. 5. Sound pressure profile at $38.6 \mathrm{~Hz}$

\subsection{Comparison with classical machine learning techniques}

To illustrate the advantages of CNN more intuitively, as shown in Fig.6, we compare the performance of our implemented $\mathrm{CNN}$ with three classical machine learning techniques: $\mathrm{k}$ nearest neighbors (KNN), Support Vector Machine (SVM), and Random forest (See the appendix for hyperparameters). We are using mean square error which helps to determine the relative accuracy of the absorption coefficients against expected values. Here, after predicting the estimated values of absorption coefficients, we further choose a threshold $\left(10^{-4}\right)$ to judge if the prediction is accurate up to certain limits or not. Hence the accuracy metric is used to estimate the effectiveness of the model to predict absorption coefficient with a certain precision. Other ML methods can have similar (or lower) MSE than the CNN, but after testing over 12 examples $\mathrm{CNN}$ seems to be the most reliable method with an average accuracy of $86 \%$. 
As shown in Fig.6, it can be clearly observed that CNN outperforms these classical techniques with an average accuracy of $86 \%$.

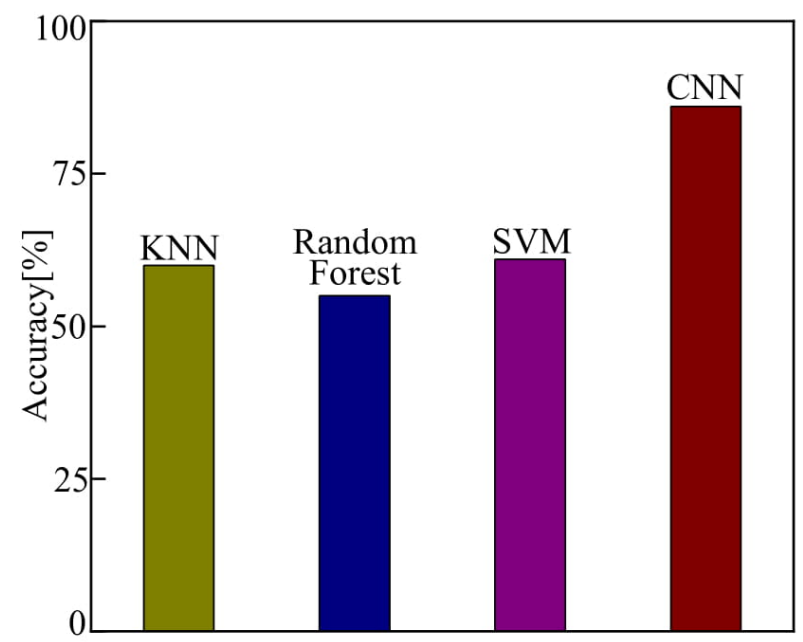

FIG. 6. The comparison between $\mathrm{CNN}$ and other classical machine learning techniques in terms of accuracy.

\section{Bandwidth improvement}

As the bandwidth is a highly desired feature when it comes to the absorption in general, and specifically in the low-frequency regime, we, in follow, introduce an approach by which we can provide broadband absorbing metasurface. Here, our objective is to design the metasurface around $38.6 \mathrm{~Hz}$ which is the lowest resonance frequency where the full absorption is achieved. For this aim, we design a supercell consisting of 25 unit-cells $(5 \times 5)$ resonating at different frequencies and having a different hole diameter of the perforated plate as shown in the inset in Fig.7b. It can be achieved by overlapping the resonances for each metasurface in the supercell. Fig.7a shows the relation between the diameter of the hole and corresponding resonance frequency for the considered designs. The diameters of the different metasurfaces are tuned between $1.5 \mathrm{~mm}$ to $4.5 \mathrm{~mm}$ to get overlapped resonances. The total thickness of the supercell is still $13 \mathrm{~mm}$, and the side length of the square supercell is $50 \mathrm{~cm}$. Here, the average absorption is higher than $95 \%$ for the given frequency range but does not reach the perfect absorption as shown in Fig. $7 \mathrm{~b}$. This is because the hybrid structure still has a small impedance mismatching, making the effective acoustic impedance at the surface slightly deviate from the impedance of the air, $\mathrm{q}_{0} \mathrm{c}_{0}$. 
(a)

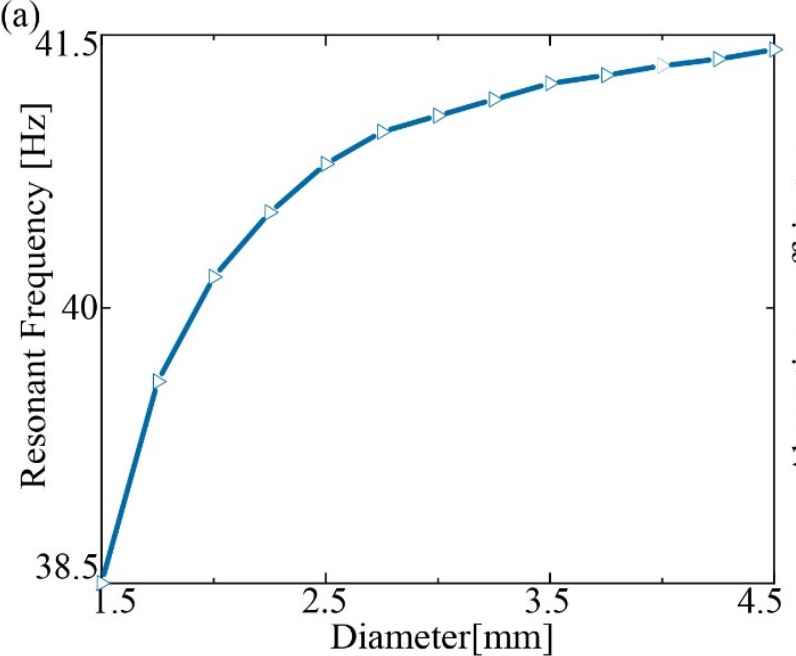

(b)

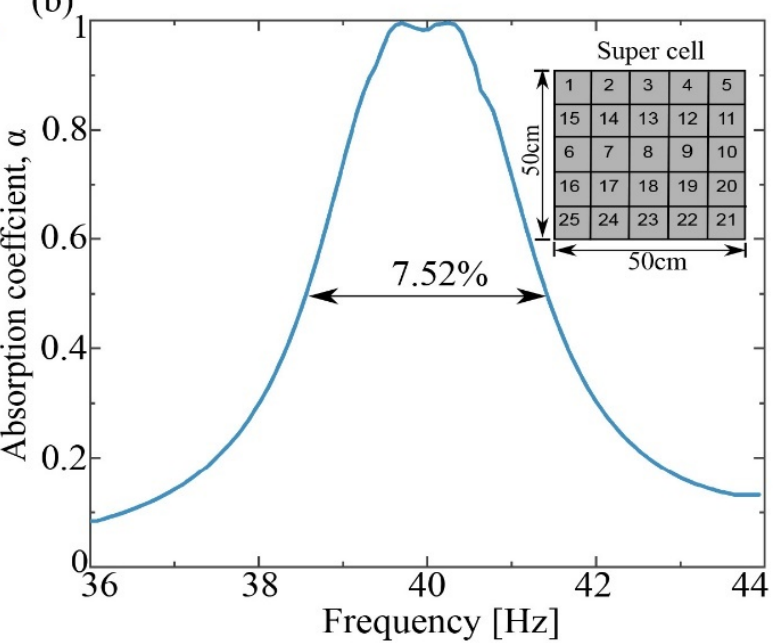

FIG. 7. (a) Relation between diameter of the holes of given metasurface and the resonant frequency (b) Bandwidth improvement using the supercell.

\section{Experiments}

To test our procedure for the application phase, we compare the experimental absorption coefficients to the ones predicted by our CNN algorithm. We use the two-microphone method as shown in Fig.8(a) to measure the acoustic absorption coefficient. The metasurface shown in Fig. 8(b), is fabricated by 3D printing based on polylactic acid (PLA) material. A lab-made impedance tube (inner size is of $10 \times 10 \mathrm{~cm}^{2}$ ), two Bruel \& Kjær 1/4-in.-diameter microphones (M1 and M2), and Bruel \& Kjær measuring module "Acoustic Material Testing" are used to measure the absorption of the metasurface [46]. The thickness of the waveguide wall is $6 \mathrm{~mm}$, and the distance between two microphones is $5 \mathrm{~cm}$. Since the operating frequency bandwidth of our measurement setup starts around $50 \mathrm{~Hz}$, we have opted to model an absorber with the $\mathrm{CNN}$ algorithm operating at $66 \mathrm{~Hz}$ to allow reliable comparison with the experimental results. We have fixed a building block at the end of the tube; the absorption spectra can be measured for the corresponding metasurface. Since, the rear of the wall is a hard wall condition, we can assume that there is no transmission. A digital signal (white noise) powered by the amplifier is sent to the loudspeaker. The absorption coefficient was obtained by analyzing the signal by two microphones. The experimental and predicted absorption curves are plotted in Fig.8(c). The obtained results show a good agreement, validating the procedure using the $\mathrm{CNN}$ program to predict acoustic absorption properties. 
(a)
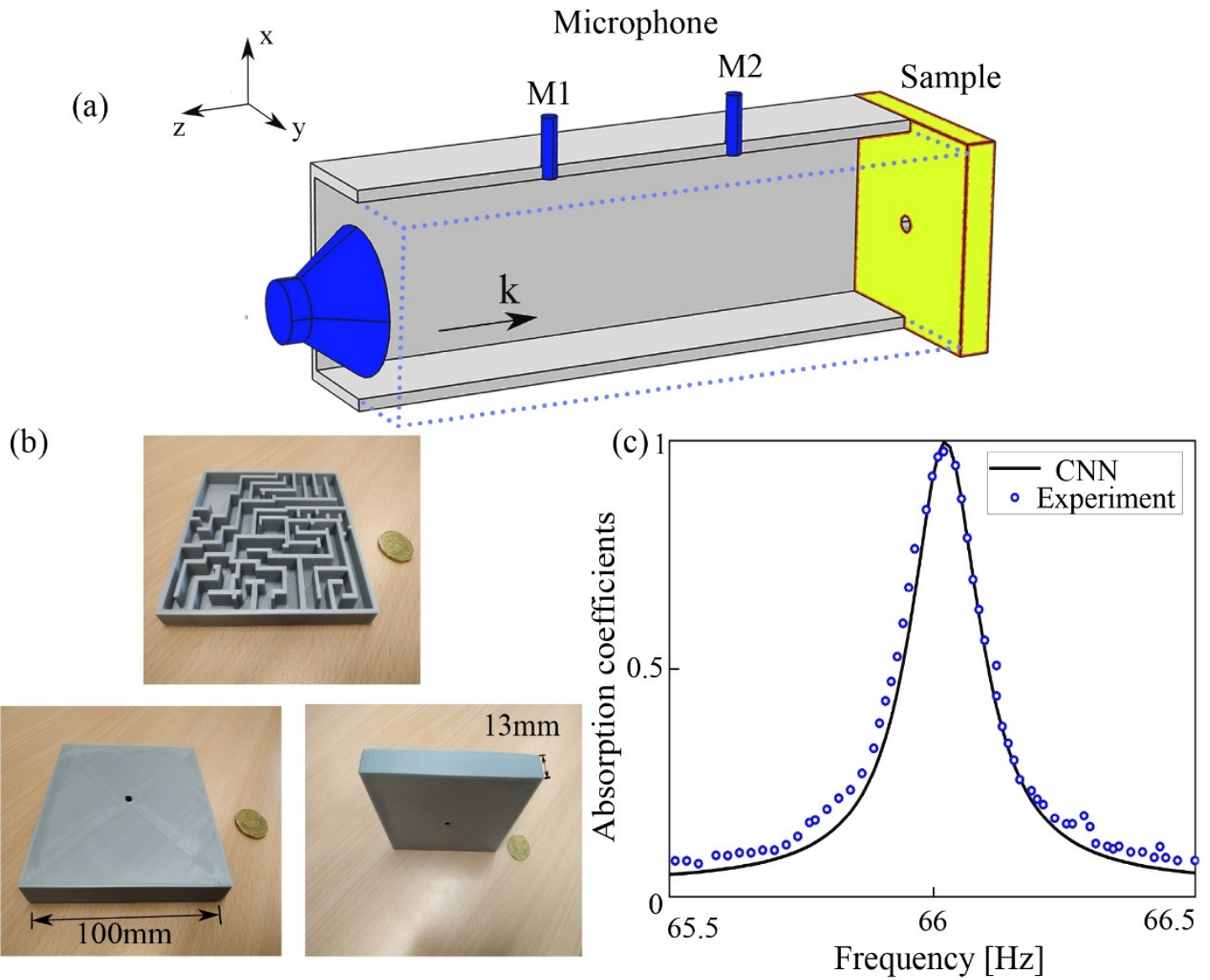

FIG. 8. (a) Schematic of the experimental setup for absorption measurement using the twomicrophone method. (b) Photographs of experimental sample with geometrical parameters: $\mathrm{a}=100 \mathrm{~mm}, \mathrm{~d}=7 \mathrm{~mm}, \mathrm{w}=\mathrm{h}+\mathrm{t}=13 \mathrm{~mm}$. (c) The absorption coefficients of the presented metasurface. The solid black line and blue dots represent the result obtained from CNN and, experimental results, respectively.

\section{Conclusion}

We have proposed a deep learning-based modeling approach for acoustic metasurface absorbers to solve the limitation of existing design methods. Accurate absorption spectrum responses of metasurface absorbers were derived using a 2D CNN-based network structure in the timescale of milliseconds, which is over four orders of magnitude faster than conventional FEM simulation software. The network predictions validated by experiments demonstrate the effectiveness of this physical mechanism and its real added value compared to the previous works. Using $\mathrm{CNN}$, an ultrathin metasurface absorber is modeled having perfect absorption at 
extremely low $38.6 \mathrm{~Hz}$ with the ultrathin thickness down to $\lambda / 684(1.3 \mathrm{~cm})$ which is the thinnest achieved metasurface ever. The total path for the propagating waves inside the channel is about $\lambda / 5.7$ which breaks the quarter-wavelength resonator theory. We envision that our method can be generically utilized for fast and accurate modeling of acoustic metasurface devices with minimum human intervention.

\section{Acknowledgement}

This work was supported by the Air Force Office of Scientific Research under Award No. FA9550-18-17021. The authors also acknowledge the support from la Region Grand Est.

\section{Data Availability}

The data that support the findings of this study are available from the corresponding author upon reasonable request.

\section{Appendix}

\section{Hyperparameters of techniques:}

CNN: We performed optimization search over the hyperparameters: number of epochs from $[10,20,50,100]$, learning rate from $[0.001,0.1,0.2,0.3]$, dropout for the dense layers from [0.0, 0.1, 0.2, 0.3], optimizer from ['SGD', 'RMSprop', 'Adagrad', 'Adadelta', 'Adam', 'Adamax'], weight initialization from ['uniform', 'normal', 'glorot_normal', 'glorot_uniform', 'he_normal', 'he_uniform']. The optimized values of hyperparameters are number of epochs 100 , learning rate is 0.2 , dropout rate is 0.0 , and weight initialization with random uniform.

Classical Machine learning techniques: For the k-nearest neighbors (KNN), we choose the number of neighbors, $n \_$neighbors $=2$. Here, the weight initialization parameter is set to the uniform. For the Support vector machine (SVM), we choose the radial basis function (RBF) kernel with the value of gamma $=0.1$ and $\mathrm{C}=100$ to initialize the model. For the random forest algorithm, we used the number of estimators, $n$ _estimators $=10$ with the default value for the maximum depth of the tree.

\section{References}

1. Fang, N., Xi, D., Xu, J., Ambati, M., Srituravanich, W., Sun, C., \& Zhang, X. Ultrasonic metamaterials with negative modulus. Nature materials, 5(6), 452-456 (2006). 
2. Ma, G., \& Sheng, P. Acoustic metamaterials: From local resonances to broad horizons. Science advances, 2(2), e1501595 (2016).

3. Yang, M., \& Sheng, P. Sound absorption structures: From porous media to acoustic metamaterials. Annual Review of Materials Research, 47, 83-114 (2017).

4. Assouar, B., Liang, B., Wu, Y., Li, Y., Cheng, J. C., \& Jing, Y. Acoustic metasurfaces. Nature Reviews Materials, 3(12), 460-472 (2018).

5. Ma, G., Yang, M., Xiao, S., Yang, Z., \& Sheng, P. X. Acoustic metasurface with hybrid resonances. Nature materials, 13(9), 873-878 (2014).

6. Cao, L., Yang, Z., Xu, Y., Fan, S., Zhu, Y., Chen, Z., Li, Y. \& Assouar, B. Flexural wave absorption by lossy gradient elastic metasurface. Journal of the Mechanics and Physics of Solids, 143, 104052 (2020).

7. Zhu, Y., Merkel, A., Donda, K., Fan, S., Cao, L., \& Assouar, B. Nonlocal acoustic metasurface for ultrabroadband sound absorption. Physical Review B, 103(6), 064102, (2021).

8. Liang, Z., \& Li, J. Extreme acoustic metamaterial by coiling up space. Physical review letters, 108(11), 114301 (2012).

9. Liu, T., Chen, F., Liang, S., Gao, H., \& Zhu, J. Subwavelength sound focusing and imaging via gradient metasurface-enabled spoof surface acoustic wave modulation. Physical Review Applied, 11(3), 034061 (2019).

10. Zhou, X., Assouar, M. B., \& Oudich, M. Acoustic superfocusing by solid phononic crystals. Applied physics letters, 105(23), 233506 (2014).

11. Li, Y., \& Assouar, B. M. Acoustic metasurface-based perfect absorber with deep subwavelength thickness. Applied Physics Letters, 108(6), 063502 (2016).

12. Huang, S., Fang, X., Wang, X., Assouar, B., Cheng, Q., \& Li, Y. Acoustic perfect absorbers via spiral metasurfaces with embedded apertures. Applied Physics Letters, 113(23), 233501 (2018).

13. Jiménez, N., Huang, W., Romero-García, V., Pagneux, V., \& Groby, J. P. Ultra-thin metamaterial for perfect and quasi-omnidirectional sound absorption. Applied Physics Letters, 109(12), 121902 (2016).

14. Romero-García, V., Theocharis, G., Richoux, O., Merkel, A., Tournat, V., \& Pagneux, V. Perfect and broadband acoustic absorption by critically coupled sub-wavelength resonators. Scientific reports, 6, 19519 (2016). 
15. Jiménez, N., Romero-García, V., Pagneux, V., \& Groby, J. P. Rainbow-trapping absorbers: Broadband, perfect and asymmetric sound absorption by subwavelength panels with ventilation. arXiv preprint arXiv:1708.03343 (2017).

16. Zhu, Y., Donda, K., Fan, S., Cao, L., \& Assouar, B. Broadband ultra-thin acoustic metasurface absorber with coiled structure. Applied Physics Express, 12(11), 114002. (2019).

17. Donda, K., Zhu, Y., Fan, S. W., Cao, L., Li, Y., \& Assouar, B. Extreme low-frequency ultrathin acoustic absorbing metasurface. Applied Physics Letters, 115(17), 173506 (2019).

18. Cummer, Steven A., Johan Christensen, and Andrea Alù. Controlling sound with acoustic metamaterials. Nature Reviews Materials 1.3: 16001 (2016).

19. Lagarrigue, C., Groby, J. P., Tournat, V., Dazel, O., \& Umnova, O. Absorption of sound by porous layers with embedded periodic arrays of resonant inclusions. The Journal of the Acoustical Society of America, 134(6), 4670-4680 (2013).

20. Wang, J., Leistner, P., \& Li, X. Prediction of sound absorption of a periodic groove structure with rectangular profile. Applied acoustics, 73(9), 960-968 (2012).

21. D'Antonio, P., \& Cox, T. Acoustic absorbers and diffusers: theory, design and application. Taylor \& Francis (2004).

22. Ivansson, S. M. Anechoic coatings obtained from two-and three-dimensional monopole resonance diffraction gratings. The Journal of the Acoustical Society of America, 131(4), 2622-2637 (2012).

23. Li, Y., Qi, S., \& Assouar, M. B. Theory of metascreen-based acoustic passive phased array. New Journal of Physics, 18(4), 043024 (2016).

24. Romero-García, V., Theocharis, G., Richoux, O., \& Pagneux, V. Use of complex frequency plane to design broadband and sub-wavelength absorbers. The Journal of the Acoustical Society of America, 139(6), 3395-3403 (2016).

25. Multiphysics, C. O. M. S. O. L. Introduction to COMSOL multiphysics ${ }^{\circledR}$. COMSOL Multiphysics, Burlington, MA (1998).

26. Gao, He, et al. "Non-blind acoustic invisibility by dual layers of homogeneous singlenegative media." Scientific Reports 7.1 (2017).

27. Miyata, K., Noguchi, Y., Yamada, T., Izui, K., \& Nishiwaki, S. Optimum design of a multi-functional acoustic metasurface using topology optimization based on Zwicker's loudness model. Computer Methods in Applied Mechanics and Engineering, 331, 116137 (2018). 
28. Noguchi, Y., Yamada, T., Otomori, M., Izui, K., \& Nishiwaki, S. An acoustic metasurface design for wave motion conversion of longitudinal waves to transverse waves using topology optimization. Applied Physics Letters, 107(22), 221909 (2015).

29. An, S., Fowler, C., Zheng, B., Shalaginov, M. Y., Tang, H., Li, H., ... \& Zhang, H. A deep learning approach for objective-driven all-dielectric metasurface design. ACS Photonics, 6(12), 3196-3207(2019).

30. Lin, X., Rivenson, Y., Yardimci, N. T., Veli, M., Luo, Y., Jarrahi, M., \& Ozcan, A. Alloptical machine learning using diffractive deep neural networks. Science, 361(6406), 1004-1008 (2018).

31. Tranter, A. D., Slatyer, H. J., Hush, M. R., Leung, A. C., Everett, J. L., Paul, K. V., ... \& Campbell, G. T. Multiparameter optimisation of a magneto-optical trap using deep learning. Nature communications, 9(1), 1-8 (2018).

32. Nadell, C. C., Huang, B., Malof, J. M., \& Padilla, W. J. Deep learning for accelerated all-dielectric metasurface design. Optics express, 27(20), 27523-27535 (2019).

33. Qiu, T., Shi, X., Wang, J., Li, Y., Qu, S., Cheng, Q., ... \& Sui, S. Deep learning: a rapid and efficient route to automatic metasurface design. Advanced Science, 6(12), 1900128. (2019).

34. Weng, J., Ding, Y., Hu, C., Zhu, X. F., Liang, B., Yang, J., \& Cheng, J. Meta-neuralnetwork for real-time and passive deep-learning-based object recognition. Nature communications, 11(1), 1-8 (2020).

35. Wan, J., Jiang, J. W., \& Park, H. S. Machine learning-based design of porous graphene with low thermal conductivity. Carbon, 157, 262-269 (2020).

36. Lusch, B., Kutz, J. N., \& Brunton, S. L. Deep learning for universal linear embeddings of nonlinear dynamics. Nature communications, 9(1), 1-10 (2018).

37. LeCun, Y., Bengio, Y., \& Hinton, G. Deep learning. nature, 521(7553), 436-444 (2015).

38. Hornik, K., Stinchcombe, M., \& White, H. Multilayer feedforward networks are universal approximators. Neural networks, 2(5), 359-366 (1989).

39. Hornik, K., Stinchcombe, M., \& White, H. Universal approximation of an unknown mapping and its derivatives using multilayer feedforward networks. Neural networks, 3(5), 551-560 (1990).

40. Cybenko, G. Approximation by superpositions of a sigmoidal function. Mathematics of control, signals and systems, 2(4), 303-314 (1989). 
41. Helmstaedter, M., Briggman, K. L., Turaga, S. C., Jain, V., Seung, H. S., \& Denk, W. Connectomic reconstruction of the inner plexiform layer in the mouse retina. Nature, 500(7461), 168-174 (2013).

42. Li, H., Fan, X., Jiao, L., Cao, W., Zhou, X., \& Wang, L. A high-performance FPGAbased accelerator for large-scale convolutional neural networks. In 26th International Conference on Field Programmable Logic and Applications (FPL) (pp. 1-9). IEEE (2016).

43. An, S., Zheng, B., Shalaginov, M. Y., Tang, H., Li, H., Zhou, L., ... \& Zhang, H. Deep learning modeling approach for metasurfaces with high degrees of freedom. Optics Express, 28(21), 31932-31942 (2020).

44. Luo, Y. T., Li, P. Q., Li, D. T., Peng, Y. G., Geng, Z. G., Xie, S. H., ... \& Zhu, X. F. (2020). Probability-Density-Based Deep Learning Paradigm for the Fuzzy Design of Functional Metastructures. Research (2020).

45. Ahmed, W. W., Farhat, M., Zhang, X., \& Wu, Y. Deterministic and probabilistic deep learning models for inverse design of broadband acoustic cloak. Physical Review Research, 3(1), 013142 (2021).

46. "Acoustics-determination of sound absorption coefficient and impedance in impedance tubes. II. Transfer-function method,” (2001), Standard No. BS ENISO, 10534-2. 